\title{
BMJ Open Quality Reasons for delay in timely administration of adjuvant chemotherapy for patients with stage III colon cancer: a multicentre cohort study from the McGill University Department of Oncology
}

\author{
Arielle Elkrief (1) , ${ }^{1}$ Genevieve Redstone, ${ }^{2}$ Luca Petruccelli, ${ }^{2}$ Alla'a Ali, ${ }^{2}$ \\ Doneal Thomas, ${ }^{2}$ Myriam Fernandez, ${ }^{2}$ Caroline Rousseau, ${ }^{2}$ Olga Aleynikova, ${ }^{3}$ \\ Dawn Anderson, ${ }^{4}$ Gabriela Ghitulescu, ${ }^{3}$ Carol-Ann Vasilevsky, ${ }^{3}$ Richard Dalfen, ${ }^{4}$ \\ Adrian Langleben, ${ }^{4}$ Sender Liberman, ${ }^{1}$ Petr Kavan, ${ }^{3}$ Thierry Alcindor ${ }^{1}$
}

To cite: Elkrief A, Redstone G, Petruccelli L, et al. Reasons for delay in timely administration of adjuvant chemotherapy for patients with stage III colon cancer: a multicentre cohort study from the McGill University Department of Oncology. BMJ Open Quality 2021;10:e000934. doi:10.1136/ bmjoq-2020-000934

AE, PK and TA contributed equally.

Received 7 February 2020 Revised 29 January 2021 Accepted 22 February 2021

Check for updates

(C) Author(s) (or their employer(s)) 2021. Re-use permitted under CC BY-NC. No commercial re-use. See rights and permissions. Published by BMJ.

${ }^{1}$ Cedar's Cancer Centre, McGill University Health Centre, Montréal, Québec, Canada ${ }^{2}$ Rossy Cancer Network, Montréal, Québec, Canada ${ }^{3}$ Segal Cancer Centre, Jewish General Hospital, Montréal,

Québec, Canada

${ }^{4}$ Department of Oncology, Saint Mary's Hospital Center, Montréal, Québec, Canada

Correspondence to Dr Arielle Elkrief;

arielle.elkrief.med@ssss.gouv. qc.ca

\section{ABSTRACT}

Purpose Adjuvant chemotherapy within 56 or 84 days following curative resection is globally accepted as the standard of care for stage III colon cancer as it has been associated with improved overall survival. Initiation of adjuvant chemotherapy within this time frame is therefore recommended by clinical practice guidelines, including the European Society for Medical Oncology. The objective of this study was to evaluate adherence to these clinical practice guidelines for patients with stage III colon cancer across the Rossy Cancer Network (RCN); a partnership of McGill University's Faculty of Medicine, McGill University Health Centre, Jewish General Hospital and St Mary's Hospital Center.

Patients and methods 187 patients who had been diagnosed with stage III colon cancer and received adjuvant chemotherapy within the RCN partner hospitals from 2012 to 2015 were included. Patient and treatment information was retrospectively determined by chart review. $X^{2}$ and Wilcoxon rank-sum tests were used to measure associations and a multivariate Cox regression model was used to determine risk factors contributing to delays in administration of adjuvant chemotherapy.

Results The median turnaround time between surgery and adjuvant chemotherapy was 69 days. Importantly, only $27 \%$ of patients met the 56 -day target, and $71 \%$ met the 84-day target. Increasing age, having more than one surgical complication and being diagnosed between 2013-2014 and 2014-2015 reduced the likelihood that patients met these targets. Furthermore, delays were observed at most intervals from surgery to first adjuvant chemotherapy treatment.

Conclusion Our study found that within these academic hospital settings, $27 \%$ of patients met the 56 -day target, and $71 \%$ met the 84 -day target. Delays were associated with hospital, surgeon and patient-related factors. Initiatives in quality improvement are needed in order to improve adherence to recommended treatment guidelines for prompt administration of adjuvant chemotherapy for stage III colon cancer.

\section{INTRODUCTION}

Colon cancer is the second most commonly diagnosed cancer in Canada. ${ }^{1}$ It is the second and third leading cause of death from cancer in men and women, respectively. ${ }^{1}$ Colon cancer prognosis is related to the stage of disease upon diagnosis. Stage III disease is defined as tumour invasion through the colonic wall extending into the submucosa or beyond with extension to the lymph nodes. ${ }^{2}$ More than one-third of patients with colon cancer present with stage III disease. ${ }^{2}$ Surgical resection is recognised as first-line treatment for localised or locally advanced colon cancer. ${ }^{2}$ Recurrence of colon cancer is thought to be secondary to micrometastases present in the circulation at the time of surgery. The goal of adjuvant chemotherapy is to eradicate these micrometastases, thereby decreasing the rate of recurrence and improving the cure rate. ${ }^{3}$ Indeed, many studies have demonstrated the benefits of postoperative adjuvant chemotherapy in patients with stage III colon cancer. ${ }^{5} \quad$ Subsequently, oxaliplatin-based chemotherapy with folinic acid, fluorouracil, oxaliplatin or capecitabine/oxaliplatin has been globally accepted as standard of care for patients with stage III colon cancer with good performance status after curative resection ${ }^{5}$ and is included in many organisations' clinical practice guidelines including the European Society for Medical Oncology (ESMO), ${ }^{7}$ Cancer Care Ontario ${ }^{8}$ and the Ministère de la Santé et des Services Sociaux (MSSS). ESMO's guidelines recommend that adjuvant chemotherapy be initiated as soon as possible, starting from 3 weeks but no later than 8-12 
weeks after surgery. ${ }^{7}$ Several non-randomised trials, metaanalyses and registry studies have investigated optimal timing and have, in general, shown that adjuvant chemotherapy administered 8 weeks after resection is associated with decreased overall survival (OS) ${ }^{9-12}$ However, while most randomised clinical trials started adjuvant chemotherapy within 6-12 weeks of resection, no randomised trial has definitively addressed optimal timing of adjuvant therapy. For this reason, we sought to evaluate adherence to both an optimal (8 weeks or 56 days) and acceptable (12 weeks or 84 days) window in which to start adjuvant chemotherapy.

In the present cohort study, we retrospectively analysed a cohort of patients with stage III colon cancer from Rossy Cancer Network (RCN)-partnered hospitals in the province of Québec to evaluate clinical practice guideline adherence for timely delivery of adjuvant chemotherapy and to determine potential reasons for delay.

\section{METHODS}

\section{Patient population}

All patients with colon cancer who were diagnosed and treated at the partner hospitals within the RCN, affiliated with McGill University between 2012 and 2015 were eligible for inclusion. Patients were excluded from the analysis if they had surgery outside of the RCN hospitals, if any had missing dates of surgery or if their chemotherapy was performed outside of the RCN-partnered hospitals (figure 1). The RCN represents a network of the largest cancer centres in Québec affiliated with McGill University: the McGill University Health Centre, the Jewish General Hospital and St Mary's Hospital Center. Founded in 2012, the RCN is a network comprised of professionals

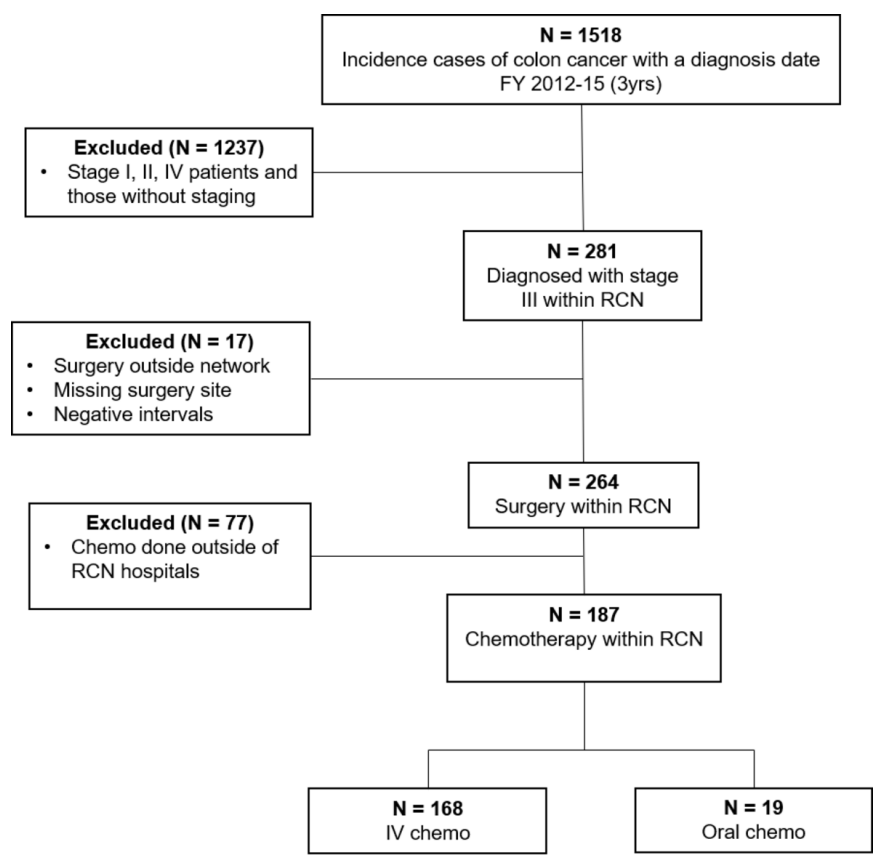

Figure 1 Patient cohort and case selection. IV, intravenous; RCN, Rossy Cancer Network. across academic and community settings and is representative of Montréal's diverse patient population. Hospitals part of the RCN see approximately 6200 new cancer cases each year of which about 850 are colorectal cancer cases. Patient data collected included age, sex, Eastern Cooperative Oncology Group (ECOG) performance status, Charlson Comorbidity Index (CCI), presence of postoperative complications, route of chemotherapy and treatment centre. Critical dates in the trajectory from surgery to start of adjuvant chemotherapy were also identified, including date of final pathology report, date of referral to medical oncology and date of first appointment with a medical oncologist.

\section{Time intervals and targets}

The most prominent time points in the trajectory of these patients were recorded: time interval 1, surgery to pathology report sign-out; time interval 2, date of pathology report to date of medical oncologist referral; time interval 3, medical oncologist referral to the first appointment; time interval 4, initial medical oncologist appointment to the start of adjuvant chemotherapy. Finally, time interval 5 , surgery to adjuvant chemotherapy delineates the overall patient time interval.

\section{Statistical analysis}

Descriptive statistics were used to describe the demographic characteristics of the patient sample. Data concerning time intervals were non-normally distributed with outliers of very long intervals. Therefore, median and IQR intervals were used in the descriptive analyses. $X^{2}$ and Wilcoxon rank-sum tests were used to assess differences in characteristics and in time intervals for patients meeting the 56-day or 84-day targets. Multivariate Cox regression analysis was used to investigate if any independent characteristics were prognostic of meeting the 56-day or 84-day target from surgery to adjuvant chemotherapy. A p-value less than 0.05 was assumed to be statistically significant. Patients with missing data were excluded from the analysis. All statistical analyses were conducted using RStudio V.3.4, an integrated development environment for R.

\section{Patient and public involvement}

This study was completed with the help of the RCN Cancer Quality Council (CQC). The CQC is an advisory committee that provides a framework for the improvement of services administered within RCN-partnered hospitals. The CQC is composed of healthcare experts across RCN-partnered hospitals and includes patient representatives from each partner hospital. Patient representatives along with other CQC members advised and participated in the design and interpretation of findings from this study.

\section{RESULTS}

\section{Patient and treatment characteristics}

Data over the 3-year study period were collected from 187 patients with resected stage III colon cancer who met our 
Table 1 Patient and treatment characteristics stratified by time-to-adjuvant chemotherapy

\begin{tabular}{|c|c|c|c|c|c|c|c|}
\hline \multirow[b]{2}{*}{ Variable } & \multirow[b]{2}{*}{ Overall } & \multicolumn{3}{|c|}{ Time-to-adjuvant chemotherapy* } & \multicolumn{3}{|c|}{ Time-to-adjuvant chemotherapy } \\
\hline & & $\leq 56$ days & $>56$ days & $P$ value & $\leq 84$ days & $>84$ days & $P$ value \\
\hline Patients (n (\%)) & 187 & $51(27)$ & 135 (73) & & $132(71)$ & $54(29)$ & \\
\hline \multicolumn{8}{|c|}{ Age at diagnosis (years) } \\
\hline Median & 70 & 65 & 72 & 0.0429 & 66 & 74 & 0.0004 \\
\hline IQR & $59-76$ & $58-73$ & $60-76$ & & $58-74$ & $63-78$ & \\
\hline Range & $36-89$ & $36-89$ & $46-87$ & & $36-89$ & $48-85$ & \\
\hline Missing (n) & 1 & & 1 & & 1 & & \\
\hline \multicolumn{8}{|l|}{$\operatorname{Sex}(n(\%))$} \\
\hline $\mathrm{F}$ & $90(48)$ & $22(43)$ & $67(50)$ & 0.5312 & $63(48)$ & $26(48)$ & 1 \\
\hline M & $97(52)$ & $29(57)$ & $68(50)$ & & $69(52)$ & $28(52)$ & \\
\hline \multicolumn{8}{|c|}{ Complications (n (\%)) } \\
\hline None & $132(72)$ & $46(90)$ & $86(66)$ & 0.0007 & $97(75)$ & 35 (66) & 0.2466 \\
\hline Yes & $50(27)$ & $4(8)$ & $45(34)$ & & $31(24)$ & $18(34)$ & \\
\hline Unknown & $1(1)$ & $1(2)$ & $0(0)$ & & $1(1)$ & $0(0)$ & \\
\hline Missing (n) & 4 & & 4 & & 3 & 1 & \\
\hline \multicolumn{8}{|l|}{ ECOG (n (\%)) } \\
\hline 0 & $64(35)$ & $20(39)$ & $44(34)$ & 0.4175 & $49(38)$ & $15(28)$ & 0.3982 \\
\hline $1-2$ & $19(10)$ & $3(6)$ & $16(12)$ & & $12(9)$ & $7(13)$ & \\
\hline Unknown & $99(54)$ & $28(55)$ & $70(54)$ & & $67(52)$ & $31(58)$ & \\
\hline Missing (n) & 5 & & 5 & & 4 & 1 & \\
\hline \multicolumn{8}{|l|}{$\mathrm{CCl}(\mathrm{n}(\%))$} \\
\hline $2-3$ & $41(22)$ & $10(20)$ & $31(23)$ & 0.5947 & $35(27)$ & $6(11)$ & 0.0208 \\
\hline $4-5$ & $91(49)$ & $29(57)$ & $61(46)$ & & $69(52)$ & $21(39)$ & \\
\hline $6-9$ & $42(23)$ & $11(22)$ & $31(23)$ & & 25 (19) & $17(31)$ & \\
\hline Unknown & $12(6)$ & $1(2)$ & $11(8)$ & & $2(2)$ & $10(19)$ & \\
\hline Missing & 1 & & 1 & & 1 & & \\
\hline \multicolumn{8}{|c|}{ Chemotherapy type (n (\%)) } \\
\hline Intravenous & $168(90)$ & $44(86)$ & $123(91)$ & 0.4837 & $120(91)$ & $47(87)$ & 0.5997 \\
\hline Oral & $19(10)$ & $7(14)$ & $12(7)$ & & $12(9)$ & $7(13)$ & \\
\hline \multicolumn{8}{|c|}{ Period of diagnosis ( $\mathrm{n}(\%))$} \\
\hline 2012-2013 & $69(37)$ & $25(49)$ & $43(32)$ & 0.0699 & $50(38)$ & $18(33)$ & 0.301 \\
\hline 2013-2014 & $64(34)$ & $16(31)$ & $48(36)$ & & $48(36)$ & $16(30)$ & \\
\hline 2014-2015 & $53(29)$ & $10(20)$ & $44(33)$ & & $34(26)$ & $20(37)$ & \\
\hline \multicolumn{8}{|c|}{ Surgeon volume ( $(\%))$} \\
\hline High & $106(58)$ & $27(54)$ & $79(60)$ & 0.5852 & $78(60)$ & $28(55)$ & 0.872 \\
\hline Low & $77(42)$ & $23(46)$ & $53(40)$ & & $53(40)$ & $23(44)$ & \\
\hline Missing (n) & 4 & 1 & 3 & & 1 & 3 & \\
\hline \multicolumn{8}{|c|}{ Surgical procedure (n (\%)) } \\
\hline Laparoscopic & $67(36)$ & $13(25)$ & $53(40)$ & 0.0699 & $46(36)$ & $20(37)$ & 0.6 \\
\hline Laparotomy & $110(60)$ & $37(73)$ & $73(55)$ & & $82(64)$ & $28(52)$ & \\
\hline Unknown & $7(4)$ & $1(2)$ & $6(5)$ & & $1(1)$ & $6(11)$ & \\
\hline Missing (n) & 3 & & 3 & & 3 & & \\
\hline \multicolumn{8}{|c|}{ Chemotherapy centre (n (\%)) } \\
\hline A & $63(34)$ & $20(39)$ & $43(32)$ & 0.2475 & $46(35)$ & $17(31)$ & 0.8644 \\
\hline B & $84(45)$ & $18(35)$ & $66(49)$ & & $58(44)$ & $26(48)$ & \\
\hline
\end{tabular}

Continued 
Table 1 Continued

\begin{tabular}{|c|c|c|c|c|c|c|c|}
\hline \multirow[b]{2}{*}{ Variable } & \multirow[b]{2}{*}{ Overall } & \multicolumn{3}{|c|}{ Time-to-adjuvant chemotherapy* } & \multicolumn{3}{|c|}{ Time-to-adjuvant chemotherapy* } \\
\hline & & $\leq \mathbf{5 6}$ days & $>56$ days & $P$ value & $\leq 84$ days & $>84$ days & P value \\
\hline $\mathrm{C}$ & $39(21)$ & $13(25)$ & $26(19)$ & & $28(21)$ & $11(20)$ & \\
\hline \multicolumn{8}{|c|}{ Same centres (n (\%)) } \\
\hline No & $17(9)$ & $3(6)$ & $14(10)$ & 0.5077 & $10(8)$ & $7(13)$ & 0.3805 \\
\hline Yes & $170(91)$ & $48(94)$ & $121(90)$ & & $122(92)$ & $47(87)$ & \\
\hline
\end{tabular}

Bold $p$-values are statistically significant from 0.05 .

${ }^{*}$ One patient had missing chemotherapy date and was excluded from the analysis.

CCl, Charlson Comorbidity Index; ECOG, Eastern Cooperative Oncology Group.

inclusion criteria. Patient characteristics for the entire cohort as well as for the patients who did not meet the 56-day or 84-day targets are shown in table 1. Median age at diagnosis was 70 years, with even distribution of men and women. ECOG status was unknown for approximately half $(54 \%)$ of the cohort. Most patients had a comorbidity CCI score of 4-5. Patients were well distributed across hospitals with the larger hospitals treating a higher percentage of patients overall (centres A and B). Univariate analysis (table 1) found a significant association between CCI and meeting the 84-day target but not the 56-day target. In a multivariate analysis (table 2), age and patients with surgical complications were found to be significantly associated with a lower likelihood of meeting the 56-day and 84-day targets, and being diagnosed in 2013-2014 or 2014-2015 was significantly associated only to the meeting the 56-day target.

\section{Interval turnaround times between surgical resection and adjuvant chemotherapy}

In general, the sequence of key events from timetosurgery to adjuvant chemotherapy was as follows: surgery, pathology report, referral to medical oncologist, first medical oncologist appointment, start of adjuvant chemotherapy (figure 2). The median turnaround time (TAT) for the entire trajectory, between surgery and adjuvant chemotherapy, was found to be approximately 69 days (table 3). Median TATs from surgery to pathology report (time interval 1) and from pathology report to medical oncologist referral (time interval 2) were 17 days and 13 days, respectively. The median time between medical oncologist referral to the first appointment was 14 days (time interval 3) and the start of chemotherapy after the first appointment occurred at a median of 19 days (time interval 4). It is important to note that not all time points were able to be captured for each case reviewed, explaining why certain time intervals were not reported for each patient.

Significant temporal differences between patients meeting and patients not meeting the 56-day target were observed in all time intervals except time interval 3 (medical oncologist referral to the first appointment). Similarly, significant temporal differences in time interval 1 (surgery to pathology report) and time interval 4 (medical oncologist appointment to treatment) were identified between patients meeting and patients not meeting the 84-day target.

\section{DISCUSSION}

This retrospective analysis of 187 patients who received adjuvant chemotherapy after resection of colon cancer demonstrated that only $27 \%$ of patients met the 56 -day target, and $71 \%$ met the 84 -day target. In multivariate analysis, presence of surgical complications and age were associated with the likelihood of not meeting the 56-day target and 84-day target, and period of diagnosis was only associated with the likelihood of not meeting the 54-day target. Our study revealed three critical intervals associated with overall delay to start of adjuvant chemotherapy (time interval 1: surgery to pathology report, time interval 2: pathology report to medical oncologist referral, and time interval 4: medical oncologist appointment to start of adjuvant chemotherapy).

The reasons for delay identified in our study aligned with findings from previous studies published on this subject. For example, one study demonstrated that in addition to postoperative complications, patient or medical oncologist-initiated delays were significant predictors in increased delay time-to-adjuvant chemotherapy. ${ }^{10}$ Patient-initiated delays included tumour board review, evaluation for clinical trials, restaging and patient's decision/acceptance of recommendation..$^{10}$ Patients with no postoperative complications and no patient/medical oncologist-initiated delays started adjuvant chemotherapy within 8.5 weeks \pm 2.1 , which may reflect the significance of delays due to referral consultations and chemotherapy booking. ${ }^{13}$ Although no studies thus far have linked surgeon volume with time-to-adjuvant chemotherapy, many studies have demonstrated the association with high surgeon volume to improved clinical outcome in patients undergoing colectomy. ${ }^{14}{ }^{15}$ Furthermore, operations performed by high-volume surgeons in high-volume hospitals had a significant decrease in postoperative complication rates and were less likely to require reoperation or transfer to the intensive care unit postoperatively. ${ }^{1415}$

Understanding the reasons for delay within the RCN is imperative because the link between timing of administration of adjuvant chemotherapy and OS in stage III 
Table 2 Multivariate model for predictors of timely adjuvant chemotherapy

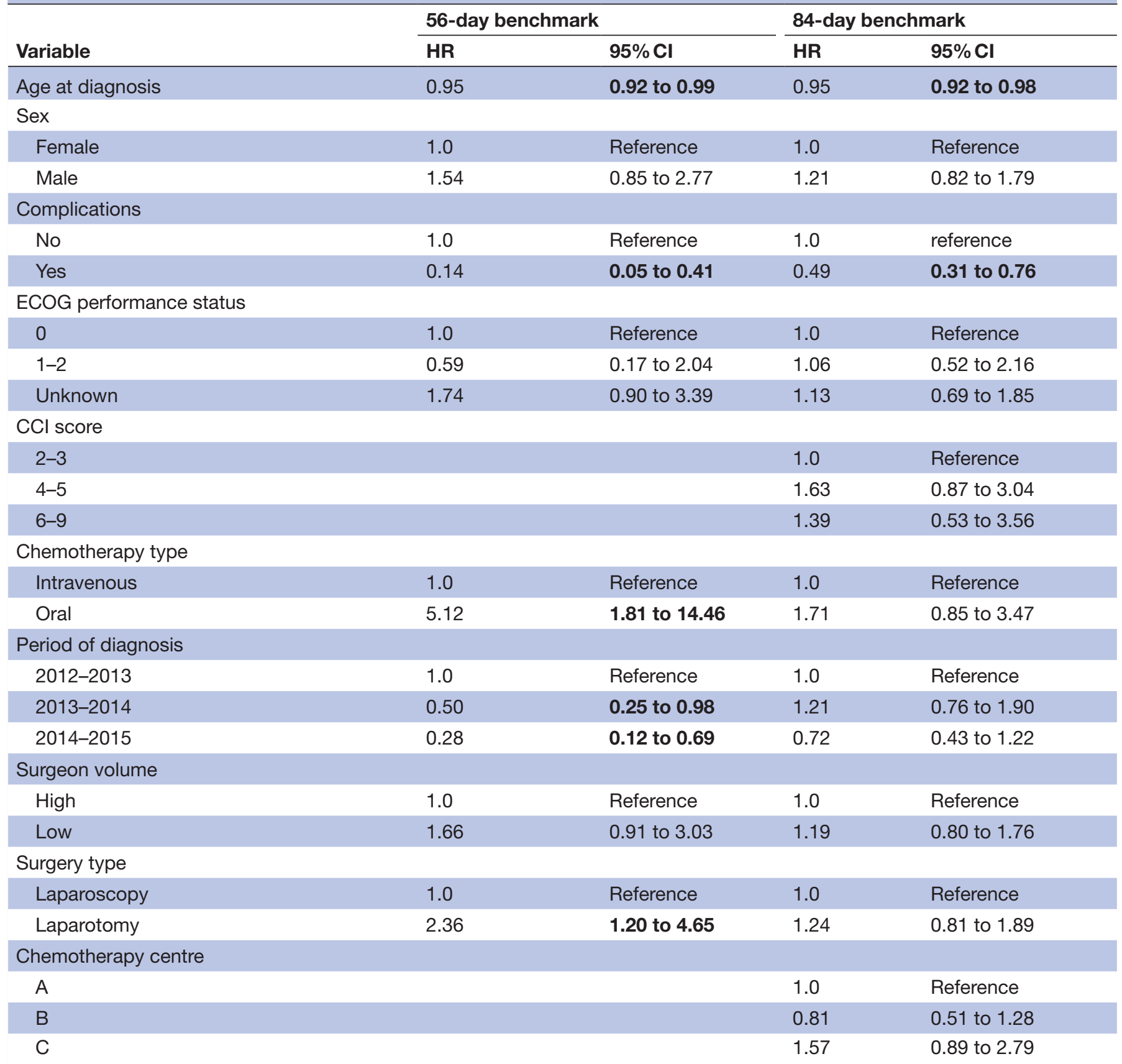

Bold estimates are significantly different from the reference group.

$\mathrm{CCl}$, Charlson Comorbidity Index; ECOG, Eastern Cooperative Oncology Group.

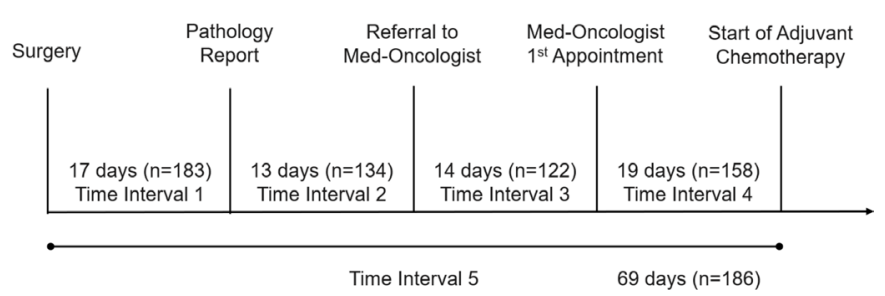

Figure 2 Patient trajectory from surgery to start of adjuvant chemotherapy. colon cancer is becoming more apparent. Indeed, two meta-analyses corroborated this association. The first meta-analysis, which included eight studies, found that delaying adjuvant chemotherapy beyond 8 weeks was associated with worse OS. ${ }^{10}$ The second meta-analysis was composed of 10 studies and demonstrated that a 4-week increase in time-to-adjuvant chemotherapy was associated with a significant decrease in both OS and disease-free survival. ${ }^{11}$ The Netherlands Comprehensive Cancer Organisation's recent analysis of patients with 
Table 3 Process time intervals in days for administration of adjuvant chemotherapy

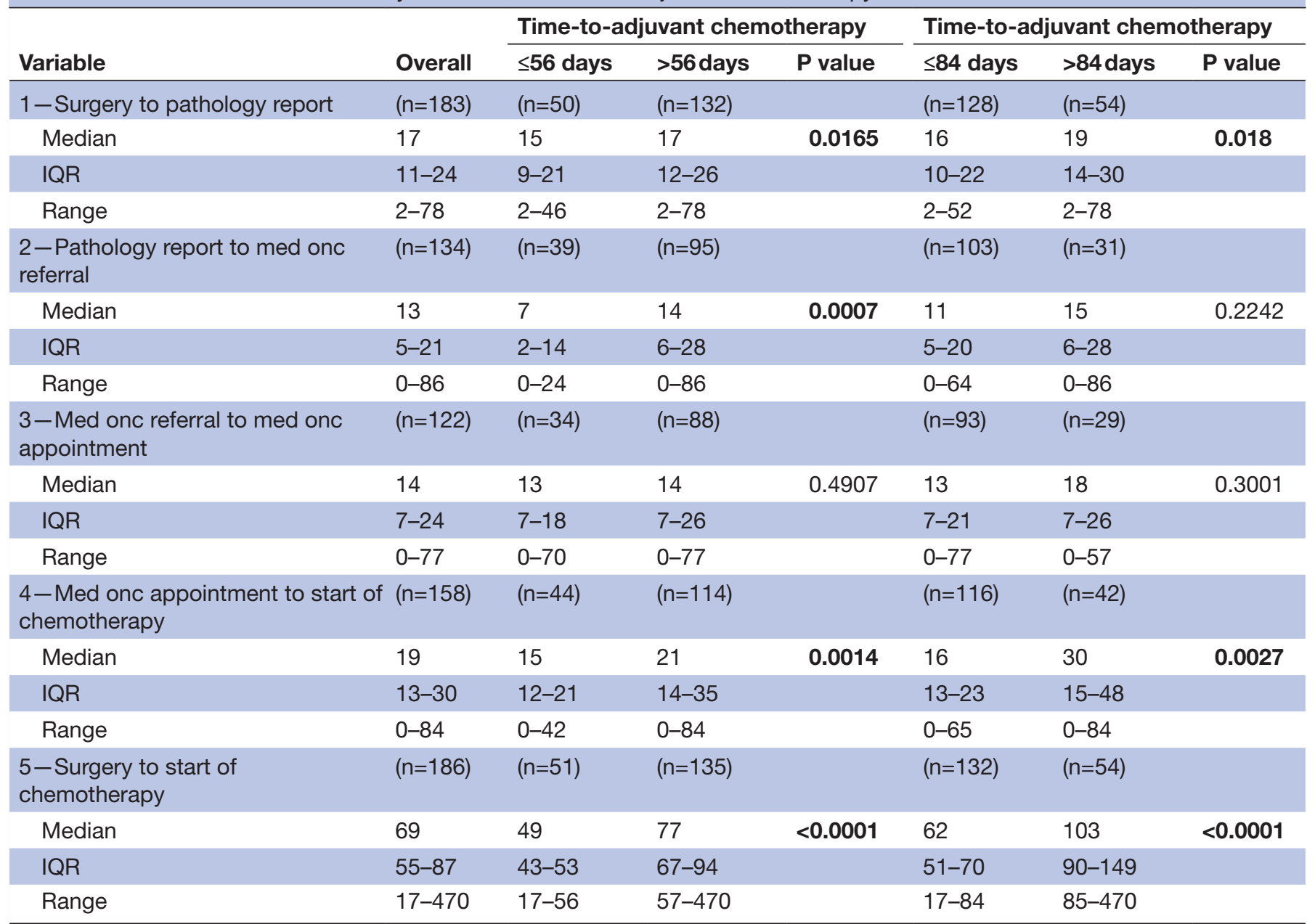

Bold estimates are significantly different from the reference group. med onc, medical oncologist.

6620 colon cancer further supports initiation of adjuvant chemotherapy within 8 weeks post-surgery, since delaying chemotherapy beyond this time frame was associated with worse OS. $^{12}$

Despite being a large, multicentre and multidisciplinary experience examining the adherence to two benchmarks (56-day and 84-day benchmarks), our retrospective study has several limitations. A significant amount of patient charts had missing ECOG performance status information, and therefore we were unable to determine whether poor ECOG performance status could impact delays. The small number of patients receiving oral chemotherapy also limited our ability to determine if the route of chemotherapy could impact delays. Furthermore, the generalisability of this study may be limited due to specific institutional reasons for delay at different centres. We also did not examine the impact of delays in chemotherapy in terms of patient clinical outcomes. Additionally, we did not analyse the potential of socioeconomic factors in influencing delays, which is a source of limitation for our study. Finally, we were not able to take into account the preference of the patient, which also may play a role in timing of initiation of adjuvant chemotherapy. Despite these limitations, our large multicentre study examined the reasons for delay for lack of adherence to two benchmarks which may be of benefit to other centres.

\section{CONCLUSIONS AND FUTURE DIRECTIONS}

Our study found that $27 \%$ of patients met the 56-day target, and $71 \%$ met the 84-day target. Delays were associated with hospital, surgeon and patient-related factors. Initiatives in quality improvement are needed in order to improve adherence to recommended treatment guidelines for prompt administration of adjuvant chemotherapy for stage III colon cancer. Following the ESMO guidelines ${ }^{7}$ and National and Regional standards brought by the MSSS in Québec, our target was defined by the RCN Gastrointestinal Disease Site Group (GI DSG). RCN's GI DSG's objective was that $85 \%$ of patients with stage III colon cancer receive adjuvant chemotherapy within 56-84days of surgery. Other benchmarks in Canada provide similar targets. ${ }^{16} 17$

Following this analysis, RCN-partnered hospitals have begun exploring the impact of select process adaptations. To reduce wait times from surgery to the first medical 
oncology appointment, surgeons now request appointments with medical oncology prior to pathology sign-out. To reduce wait times from the first medical oncology appointment to neoadjuvant chemotherapy, medical oncologists now consult with patients while they recover from surgery regardless of complications. Involving a multidisciplinary oncology team on diagnosis may also reduce delay times. Finally, a prioritisation committee was created to inform centralised booking services of priority patients. Continued prospective monitoring of TATs is essential and will reveal the extent to which the process adaptations improve guideline adherence.

Twitter Arielle Elkrief @arielleelkriefMD

Acknowledgements The authors would like to thank Warren Sateran for his work on the initial data management plan.

Contributors PK and TA conceived the original idea for the study. TA and PK supervised the completion of the study and manuscript. AE conducted chart review and designed the study. AE, GR and LP contributed equally to redaction and editing of the manuscript and data interpretation. DT was responsible for statistical analysis. All authors contributed to editing of the manuscript.

Funding This work was funded with generous support from the Rossy Cancer Network.

Competing interests None declared.

Patient consent for publication Not required.

Ethics approval The Director of Professional Services of our institution approved this study, letter attached in supplemental section. As per institutional policy, ethics approval is not required for quality improvement studies, and therefore chart review permission is granted by the Director of Professional Services. After chart review, the resulting data set was fully anonymised for analysis.

Provenance and peer review Not commissioned; externally peer reviewed.

Data availability statement Data are available upon reasonable request. Deidentified patient data are available upon request, please contact the Rossy Cancer Network at rcn@mcgill.ca.

Supplemental material This content has been supplied by the author(s). It has not been vetted by BMJ Publishing Group Limited (BMJ) and may not have been peer-reviewed. Any opinions or recommendations discussed are solely those of the author(s) and are not endorsed by BMJ. BMJ disclaims all liability and responsibility arising from any reliance placed on the content. Where the content includes any translated material, BMJ does not warrant the accuracy and reliability of the translations (including but not limited to local regulations, clinical guidelines, terminology, drug names and drug dosages), and is not responsible for any error and/or omissions arising from translation and adaptation or otherwise.

Open access This is an open access article distributed in accordance with the Creative Commons Attribution Non Commercial (CC BY-NC 4.0) license, which permits others to distribute, remix, adapt, build upon this work non-commercially, and license their derivative works on different terms, provided the original work is properly cited, appropriate credit is given, any changes made indicated, and the use is non-commercial. See: http://creativecommons.org/licenses/by-nc/4.0/.
ORCID iD

Arielle Elkrief http://orcid.org/0000-0003-1981-1535

\section{REFERENCES}

1 Public Health Agency of Canada, Statistics Canada, Canadian Cancer Society, et al. Release notice - Canadian Cancer Statistics 2019. Health Promot Chronic Dis Prev Can 2019;39:255.

2 Benson AB, Bekaii-Saab T, Chan E, et al. Localized colon cancer, version 3.2013: featured updates to the NCCN guidelines. J Natl Compr Canc Netw 2013;11:519-28.

3 Sargent D, Sobrero A, Grothey A, et al. Evidence for cure by adjuvant therapy in colon cancer: observations based on individual patient data from 20,898 patients on 18 randomized trials. J Clin Oncol 2009;27:872-7.

4 Shah MA, Renfro LA, Allegra CJ, et al. Impact of patient factors on recurrence risk and time dependency of oxaliplatin benefit in patients with colon cancer: analysis from Modern-Era adjuvant studies in the adjuvant colon cancer end points (accent) database. J Clin Oncol 2016;34:843-53.

5 Czaykowski PM, Gill S, Kennecke HF, et al. Adjuvant chemotherapy for stage III colon cancer: does timing matter? Dis Colon Rectum 2011;54:1082-9.

6 Babaei M, Balavarca Y, Jansen L, et al. Administration of adjuvant chemotherapy for stage II-III colon cancer patients: an European population-based study. Int J Cancer 2018;142:1480-9.

7 Schmoll HJ, Van Cutsem E, Stein A, et al. ESMO consensus guidelines for management of patients with colon and rectal cancer. A personalized approach to clinical decision making. Ann Oncol 2012;23:2479-516.

8 Meyers BM, Cosby R, Quereshy F, et al. Adjuvant chemotherapy for stage II and III colon cancer following complete resection: a cancer care Ontario systematic review. Clin Oncol 2017;29:459-65.

9 Klein M, Azaquoun N, Jensen BV, et al. Improved survival with early adjuvant chemotherapy after colonic resection for stage III colonic cancer: a nationwide study. J Surg Oncol 2015;112:538-43.

10 Wasserman DW, Boulos M, Hopman WM, et al. Reasons for delay in time to initiation of adjuvant chemotherapy for colon cancer. $J$ Oncol Pract 2015;11:e28-35.

11 Biagi JJ, Raphael MJ, Mackillop WJ, et al. Association between time to initiation of adjuvant chemotherapy and survival in colorectal cancer: a systematic review and meta-analysis. JAMA 2011;305:2335-42.

12 Bos ACRK, van Erning FN, van Gestel YRBM, et al. Timing of adjuvant chemotherapy and its relation to survival among patients with stage III colon cancer. Eur J Cancer 2015;51:2553-61.

13 Chan A, Woods R, Kennecke $\mathrm{H}$, et al. Factors associated with delayed time to adjuvant chemotherapy in stage III colon cancer. Curr Oncol 2014;21:181-6.

14 Damle RN, Macomber CW, Flahive JM, et al. Surgeon volume and elective resection for colon cancer: an analysis of outcomes and use of laparoscopy. J Am Coll Surg 2014;218:1223-30.

15 Huo YR, Phan K, Morris DL, et al. Systematic review and a metaanalysis of hospital and surgeon volume/outcome relationships in colorectal cancer surgery. J Gastrointest Oncol 2017;8:534-46.

16 Webster TM, Newell C, Amrhein JF, et al. Cancer care Ontario guidelines for radical prostatectomy: striving for continuous quality improvement in community practice. Can Urol Assoc J 2012;6:442-5.

17 Lima ISF, Yasui Y, Scarfe A, et al. Association between receipt and timing of adjuvant chemotherapy and survival for patients with stage III colon cancer in Alberta, Canada. Cancer 2011;117:3833-40. 